\title{
Kinematic Geometry of Osteotomies
}

\author{
Erin J. Smith ${ }^{1}$, J. Tim Bryant ${ }^{1,3}$, and Randy E. Ellis ${ }^{1,2,3}$ \\ 1 Mechanical Engineering, Queen's University at Kingston, Canada K7L 3N6 \\ 2 School of Computing, Queen's University at Kingston, Canada K7L 3N6 \\ 3 Department of Surgery, Queen's University at Kingston, Canada K7L 3N6
}

\begin{abstract}
This paper presents a novel method for defining an osteotomy that can be used to represent all types of osteotomy procedures. In essence, we model an osteotomy as a lower-pair mechanical joint to derive the kinematic geometry of the osteotomy. This method was implemented using a commercially available animation software suite in order to simulate a variety of osteotomy procedures. Two osteotomy procedures are presented for a femoral malunion in order to demonstrate the advantages of our kinematic model in developing optimal osteotomy plans. The benefits of this kinematic model include the ability to evaluate the effects of various kinds of osteotomy and the elimination of potentially errorprone radiographic assessment of deformities.
\end{abstract}

\section{Introduction}

An osteotomy, the surgical cutting of bone, is used in many orthopedic procedures for the purpose of correcting mechanical and aesthetical deformities of bones and joints. Bone deformities may arise from many causes including congenital defects, poor nutrition, disease, and post-traumatic healing. These deformities can be described by two elemental deformities, in rotation and in translation. It is common to further subdivide these into rotation and translation about/along the long axis of a bone, and rotation and translation about/along some other (transverse) axis. Simple deformities consist of only one elemental deformity, whereas complex deformities are a combination of simple deformities. These deformities are illustrated through the use of an elongated cuboid in Fig.1.

Numerous osteotomy procedures have been described in the orthopedics literature, differing according to the anatomical site of the osteotomy, orientation and geometry of the cut, and the type of movement of the osteotomized segments [1]. Common osteotomy techniques include rotational, transverse displacement, longitudinal displacement, wedge, and oblique osteotomies. A variety of osteotomy plane geometries, such as Z-cut, step-cut and chevron, have been developed in order to improve the surface contact of the bone segments in some of these procedures.

Typically, radiographic and CT images are used to identify orthopedic deformities and select an appropriate osteotomy procedure. Computer-assisted osteotomy planning has, to date, targeted a specific type of osteotomy procedure [2, 3, 4, 5, 6, 6, 7, 8]. Unfortunately, these methods do not allow surgeons to readily compare the results of using different procedures to correct a deformity and further require that surgeons be familiar with a number of software programs.

This paper presents a novel method for defining an osteotomy procedure that can be used to represent all types of osteotomy. This method can be readily incorporated into 


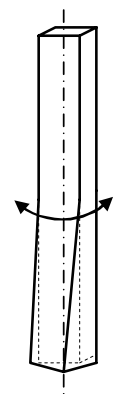

$\mathbf{a}$

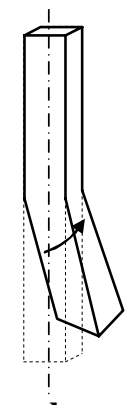

b

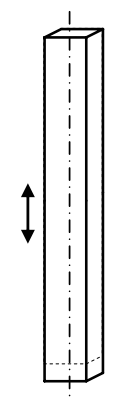

c
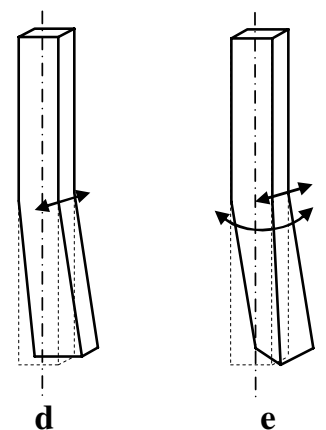

Fig. 1. Common orthopedic deformities: (a) rotational deformation about the long axis; (b) angular deformation about a transverse axis; (c) translational deformation along the long axis; (d) translational deformation along a transverse axis; and (e) complex, both rotational and translational

computer graphics in order to simulate a variety of osteotomy procedures. In essence, we model an osteotomy as a lower-pair mechanical joint to derive the kinematic geometry of the procedure.

We were inspired to consider kinematic geometry because of a common requirement of mechanical joints and the healing of an osteotomy: surface congruence. Bearing surfaces must be congruent; otherwise, excessive wear or catastrophic failure may result. For bone to heal, the surfaces of the osteotomized bone should be [roughly] congruent, both for weight-bearing resistance and because mechanical strain is a necessary element in bone remodeling.

\section{Kinematic Representation of an Osteotomy}

An osteotomy permits relative 3D motion of the osteotomized bone segments. If one segment is selected as the base segment, the other segment has up to six degrees of freedom of relative motion: three translations and three rotations. Simple representations of relative motion, such as homogeneous coordinate transformations, are commonly used in computer graphics and robotics [9]. It is straightforward to represent any given type of osteotomy by such a transformation; this was reported for the oblique osteotomy as early as 1952 [10] and as recently as 2005 [11]. However, these general representations do not elegantly capture the relative motion induced by a particular osteotomy.

The literature on robotics and mechanism theory is rich with descriptions of relative motion of rigid members [12]. Because any particular osteotomy procedure restricts the transformation allowed between the bone segments, it may be useful to consider the osteotomy as a virtual joint.

\subsection{A Virtual-Joint Model}

In robotics, joints connect successive links and constrain the motion that can occur between the links. The number of degrees of freedom of a joint is known as the joint 


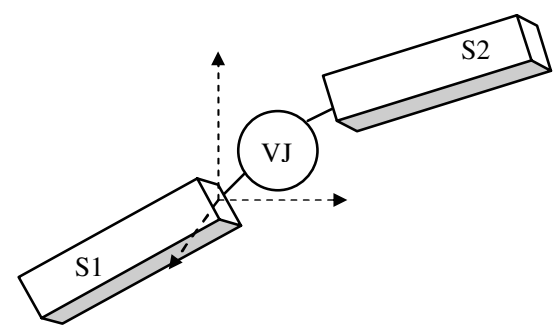

$\mathbf{a}$

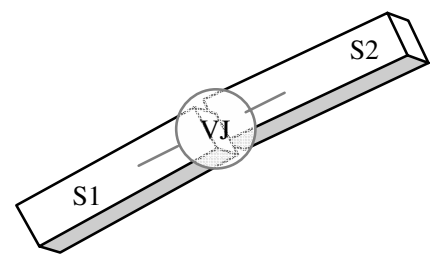

b

Fig. 2. Virtual-joint model of an osteotomy. (a) A virtual joint (VJ) connects the osteotomized segments, S1 and S2. Motion of S2 can be defined with respect to a coordinate frame attached to $\mathrm{S} 1$ at the position of the osteotomy site, having the same orientation as the joint axes; (b) The joint is not a physical presence as it is in robotic systems and, therefore, does not actually compromise the proximity of the osteotomized surfaces as the graphical representation in (a) may suggest.

mobility $m$. In real mechanisms the mobility can take on values of $0<m \leq 6$. The simplest joint has a joint mobility of $m=1$, allowing movement in a single direction only; in robotics the common mobility-one joints are either prismatic (translation only in a fixed direction) or revolute (rotation only about a fixed axis). Complex joints consisting of higher degrees of freedom can be made by combining revolute and prismatic joints, and allow motion in multiple directions.

A distinction made early in the twentieth century divided joint kinematics into lower-pair and higher-pair motions [13]. A kinematic higher pair has non-congruent joint surfaces; in the real world, such a bearing surface is not durable. An example of a higher pair is a point loading of a plane, for which transverse motion would either wear the point to a locally planar surface or break the point.

In general there are six classes of kinematic lower pairs, each with a specify type of movement: prismatic, revolute, helical 11, cylindrical, planar, and spherical. If the osteotomized bone surfaces are congruent or nearly so then it must be the case that, for any given type of osteotomy, the relative motion between the bone segments is constrained to be a kinematic lower pair.

We have found a direct mapping from kinematic lower pairs to common osteotomy procedures, which we call virtual joints (VJs). A VJ is a kinematic constraint on the relative segment motion, shown in Fig. 2. A VJ has two important uses in planning an osteotomy. First, a planning system can use a VJ to restrict the allowable motion of bone models so that a surgeon can determine beforehand the effects of various kinds of osteotomy. Second, once a specific osteotomy is selected, the congruent surfaces of the corresponding kinematic lower pair can be calculated and provided to the surgeon for intraoperative guidance of the osteotomy procedure.

We have searched the orthopedic literature extensively and categorized osteotomy procedures as various kinds of VJs. As shown in Table 1 the kinds of osteotomy used to correct simple deformities are either prismatic or revolute VJs, each with mobility

\footnotetext{
${ }^{1}$ Although helical joints are useful for screws, nuts, bolts, etc., they seem to have little utility in describing osteotomy.
} 
Table 1. Osteotomy procedures modeled as virtual $m=1$ lower-pair joints

\begin{tabular}{|c|l|c|c|}
\hline Osteotomy & \multicolumn{1}{c|}{ Common Examples } \\
$\begin{array}{c}\text { Transverse } \\
\text { Rotational }\end{array}$ & $\begin{array}{l}\text { Rotational/derotational osteotomy; } \\
\text { Transtrochanteric rotational osteotomy of } \\
\text { the femoral head; Rotational acetabular } \\
\text { osteotomy }\end{array}$ \\
\hline $\begin{array}{c}\text { Closing } \\
\text { Wedge }\end{array}$ & $\begin{array}{l}\text { Coventry high tibial osteotomy; } \\
\text { varus/valgus correction }\end{array}$ \\
\hline $\begin{array}{c}\text { Opening } \\
\text { Wedge }\end{array}$ & $\begin{array}{l}\text { High tibial opening wedge osteotomy; } \\
\text { varus/valgus correction; Distal radius } \\
\text { osteotomy }\end{array}$ \\
\hline $\begin{array}{c}\text { Oblique } \\
\text { Tibial/femoral malunions }\end{array}$ \\
$\begin{array}{c}\text { Longitudinal } \\
\text { Displacement }\end{array}$ & $\begin{array}{l}\text { Lengthening/shortening of long bones } \\
\text { Transverse } \\
\text { Displacement }\end{array}$ & $\begin{array}{l}\text { Pelvic osteotomy; Hallux valgus } \\
\text { correction }\end{array}$
\end{tabular}

Table 2. Use of $m \geq 2$ lower-pair joints in describing osteotomy

\begin{tabular}{|l|l|}
\hline \multicolumn{1}{|c|}{ Osteotomy } & VJ Model \\
\hline $\begin{array}{l}\text { Combined wedge and transverse displacement } \\
\text { osteotomy; Combined longitudinal displacement and } \\
\text { rotational osteotomy }\end{array}$ \\
\hline $\begin{array}{l}\text { Combined rotational and transverse displacement } \\
\text { osteotomy }\end{array}$
\end{tabular}

$m=1$. VJs of higher mobility, $m \geq 2$, can be used to model osteotomy procedures that correct complex deformities, and are shown in Table 2 .

\section{Implementation and Assessment}

The VJ model can be applied to the computer-assisted planning of an osteotomy by creating constraints that define how computer images can be moved relative to one another. Commercial computer-graphics animation software (Maya 6 Complete, Alias/ Wavefront, Toronto, Canada) was used to implement VJs. This software package was 
selected because of its powerful graphics and development capabilities. Algorithms were developed to constrain motion for all five lower-pair joints of the VJ model, and both forward and inverse planning techniques were implemented.

Virtual osteotomy simulations were conducted using 3D surface meshes of a deformed bone and a normal counterpart. These surface meshes were obtained by laserscanning foam models of bones (Pacific Research laboratories, Bellinham, USA). Osteotomy simulations were performed on bone models having both simple and complex deformities. For brevity, only two VJ osteotomies will be presented here.

\subsection{Forward Planning: An Opening-Wedge Osteotomy}

The forward virtual-osteotomy process simulates a surgical procedure. Consider, for example, an opening-wedge osteotomy of a malunited femur with a 30-degree angulation deformity modeled using a revolute virtual joint. This osteotomy was simulated in four steps:

1. The normal and deformed surface meshes were manually aligned in order to visualize the deformity in 3 dimensions, shown in Fig 3 (a);

2. A graphical plane was used to define the position and orientation of the osteotomy, shown in Fig 3 (b). The coordinate axes of the plane were used to indicate the orientation of the $\mathrm{VJ}$ axis;

3. The surface mesh of the deformed bone was separated to create two independent meshes along the osteotomy plane; and

4. A revolute joint constraint was applied to the osteotomized bone which restricted the movement of the mesh images to rotation about the joint axis. A graphical user interface facilitated movement of the bone image according to the constraint model, shown in Fig[3 (c). A separate user interface element was created for each degree of freedom depending on the type of joint constraint.

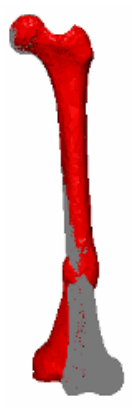

$\mathbf{a}$

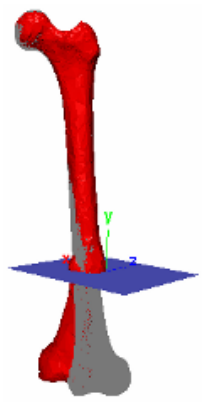

b

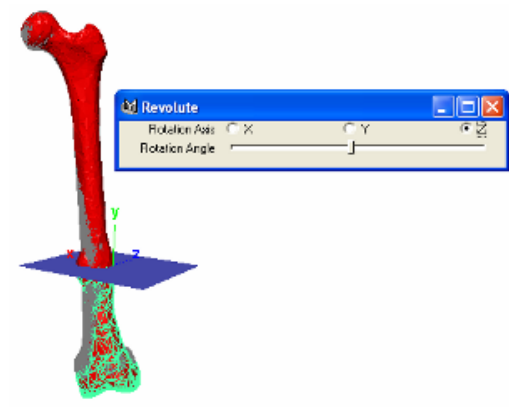

c

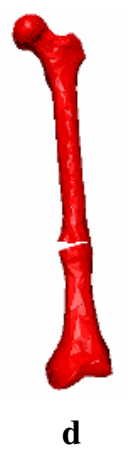

d

Fig. 3. Virtual opening-wedge osteotomy: (a) Alignment of normal and deformed surface meshes; (b) A graphical plane was used to specify the location of the osteotomy; (c) A slider interface was used to adjust the rotation about the joint axis; (d) Result of the opening-wedge osteotomy 


\subsection{Inverse Planning: An Oblique Osteotomy}

An oblique, single-cut, osteotomy can be used to correct simple angulation deformities as well as complex deformities involving rotational and angular elements. The surgeon makes a single cut such that rotation in the plane of the cut corrects the deformity. The oblique osteotomy, when indicated, is preferred over a wedge osteotomy because it eliminates changes in bone length due to the removal or addition of a wedge, and results in a stable interface for fixation.

Although the theory of the oblique plane is well established [10], determining the orientation of the plane can be quite difficult. Efforts have been made to determine the orientation of the oblique plane from mathematical formulas based on angles of the deformity measured from radiographs [14]. As as extension of these formulas, lookup tables were developed to facilitate the mathematical calculations [15]. Quite recently, a mechanical device was constructed to determine the orientation of the oblique plane [11]; interestingly, these authors noted that the use of a computer would be ideally suited to solving this problem.

An inverse planning algorithm was implemented to determine the orientation of the oblique osteotomy plane. The process was the same as that described for the forward virtual-joint osteotomy; The osteotomy was modeled as a spherical joint constraint, and the user manipulated the spherical degrees of freedom to correct the deformity. The result of the rotational transformation was used to determine the orientation of the oblique osteotomy plane using an equivalent angle-axis representation. Test models were created to validate the algorithm, comparing the orientation of the oblique plane to the results obtained by Sangeorzan et al. [14, 15].

The inverse planning algorithm was used to determine the orientation of the oblique plane for a malunited femur with 30-degree angulation, presented earlier as an openingwedge osteotomy (Fig. 44).

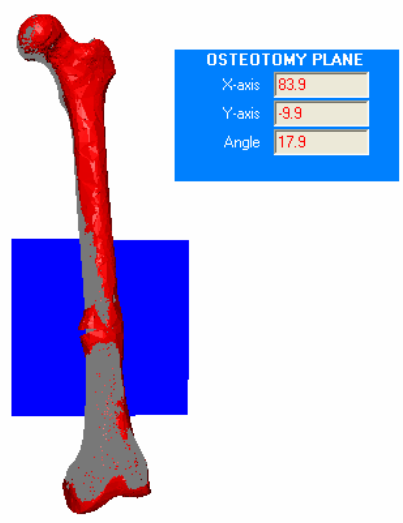

Fig. 4. Virtual osteotomy of a femur using the inverse model to determine the orientation of the osteotomy plane. The display window indicates the orientation of the oblique plane and the rotation angle about the equivalent rotation axis. In this case, the oblique plane that corrects the deformity is not clinically feasible since it would result in an extremely oblique and long osteotomy plane. 


\section{Discussion}

The VJ model provides the mathematical foundation for a novel computer-based preoperative osteotomy planner with the ability to represent all types of osteotomy procedures. By using the VJ method, multiple osteotomy techniques can be evaluated in order to select an optimal plan. For the examples presented, it is possible to compare the use of an oblique plane (that would not completely correct the deformity) to an opening-wedge osteotomy (that may involve leg-lengthening).

A clear advantage of the $\mathrm{VJ}$ model is the elimination to initially characterize orthopedic deformities in terms of radiographic measurements. In the $\mathrm{VJ}$ model, representation of the osteotomy is directly related to the relative motion that occurs between the osteotomized segments instead of the nature of the deformity itself. This novel approach is especially advantageous in determining the orientation of an oblique osteotomy plane since existing methods involving mathematical formulas [14], look-up tables [15] and a mechanical device [11] rely on initial radiographic assessment, and limit the ability to create optimal preoperative plans. A direct transformation-based solution can increase the accuracy of preoperative osteotomy plans by eliminating errors associated with radiographic measurements that may not be perfectly aligned to the anatomical planes from which the deformities are referenced. A transformation-based approach also allows surgeons to experiment with the outcome of the oblique procedure to produce an optimal plan with both feasible geometry and sufficient deformity correction. It is difficult to do this with the existing approaches, as the relationship between the parameters used to represent the deformity and the orientation of the osteotomy plane is not straightforward.

The accuracy of the current implementation is dependent on the alignment of the deformed bone with its normal counterpart. In this implementation, alignment was performed manually; however, the authors recognize that automated registration techniques could be used for alignment.

For simplicity, the VJ model was used here to implement only those osteotomy procedures that could be represented by planar cuts and motion defined by a single kinematic lower pair. It should be noted, however, that the fundamentals of the VJ model can be easily extended to represent osteotomy procedures with more complex geometries and motions. For instance, the Z-cut, chevron, and crescentic osteotomy could be implemented by creating a library of osteotomy geometries to supplement the lower-pair-joint constraints. The effects of external fixators, such as Taylor and Ilivarov frames, could be including by considering systems of kinematic lower pairs. Procedures with complex motion patterns can be incorporated into the model by extending the library of virtual constraints.

\section{Conclusion}

A novel method for representing an osteotomy as a constrained transformation was presented. This method was used to simulate a number of osteotomy procedures whose kinematic geometry can be described by a lower pair joint. The proposed virtual-joint model has the capability to represent any type of osteotomy procedure. Potential advantages of this method include the ability to evaluate the effects of various kinds of 
osteotomy and the elimination of potentially error-prone radiographic assessment of deformities. The VJ model provides an improved means to define optimal preoperative plans that can be used intraoperatively to guide the osteotomy procedure.

\section{Acknowledgements}

This research was supported in part by the Natural Sciences and Engineering Research Council of Canada, the Institute for Robotics and Intelligent Systems, Communications and Information Technology Ontario, and the Ontario Research and Development Challenge Fund.

\section{References}

[1] Canale, S.T., ed.: Campbell's Operative Orthopaedics Tenth Edition. Mosby Inc., Philadelphia PA (2003)

[2] Bilic, R., Zdravkovic, V., Boljevic, Z.: Osteotomy for deformity of the radius: Computerassisted three-dimensional modelling. Journal of Bone and Joint Surgery 76 (1994) 150 154

[3] Moctezuma, J.L., Gossé, F., Schulz, H.J.: A computer and robot aided surgery system for accomplishing osteotomies. In: International Symposium on Medical Robotics and Computer Assist ed Surgery. (1994) 31-35

[4] Chao, E.Y.S., Sim, F.H.: Computer-aided preoperative planning in knee osteotomy. Iowa Orthopaedic Journal 15 (1995) 4-18

[5] Ellis, R.E., Tso, C.Y., Rudan, J.F., Harrison, M.M.: A surgical planning and guidance system for high tibial osteotomy. Computer Aided Surgery 4 (1999) 264-274

[6] Croitoru, H., Ellis, R.E., Small, C.F., Prihar, R., Pichora, D.R.: Fixation-based surgery: A new technique for distal radius osteotomy. Computer Aided Surgery 6 (2001) 160-169

[7] Mayman, D., Rudan, J.F., Yach, J., Ellis, R.E.: The Kingston periacetabular osteotomy: A new computer-enhanced technique. Computer Aided Surgery 7 (2002) 179-186

[8] Athwal, G.S., Ellis, R.E., Small, C.F., Pichora, D.R.: Outcomes of computer-assisted distal radius osteotomy. Journal of Hand Surgery 28 (2003) 951-958

[9] Craig, J.J.: Introduction to Robotics: Mechanics and Control. Addison-Wesley, Reading MA (1989)

[10] d'Aubigne, R.M., Descamps, L.: L'osteotomie plane oblique dans la correction des deformations des membres. Memoires: Academie de Chirurgie 78 (1952) 271-276

[11] Meyer, D., Siebenrock, K., Schiele, B., Gerber, C.: A new methodology for the planning of single-cut corrective osteotomies of mal-aligned bones. Clinical Biomechanics 20 (2005) 223-227

[12] McCarthy, J.M.: An Introduction to Theoretical Kinematics. MIT Press, Cambridge MA (1990)

[13] Hunt, K.H.: Kinematic Geometry of Mechanisms. Oxforn University Press, New York (1990)

[14] Sangeorzan, B.P., Judd, R., Sangeorzan, B.J.: Mathematical analysis of single-cut osteotomy for complex long bone deformity. Journal of Biomechanics 22 (1989) 1271-1278

[15] Sangeorzan, B.P., Sangeorzan, B.J., Hansen, S., Judd, R.: Mathematically directed singlecut osteotomy for correction of tibial malunion. Journal of Orthopaedic Trauma 3 (1989) 267-275 